\title{
Pars plana vitrectomy for diabetic macular edema. Internal limiting membrane delamination vs posterior hyaloid removal. A prospective randomized trial
}

\author{
Hans Hoerauf • Anne Brüggemann • Manuela Muecke • \\ Julia Lüke • Maya Müller • Einar Stefánsson • \\ Hans-Peter Hammes • Claudia Weiß
}

Received: 25 October 2010/Revised: 26 December 2010 /Accepted: 29 December 2010 /Published online: 18 January 2011

(C) The Author(s) 2011. This article is published with open access at Springerlink.com

\begin{abstract}
Background Diabetes mellitus, as well as subsequent ocular complications such as cystoid macular edema (CME), are of fundametal socio-economic relevance. Therefore, we evaluated the influence of internal limiting membrane (ILM) removal on longterm morphological and functional outcome in patients with diabetes mellitus (DM) type 2 and chronic CME without evident vitreomacular traction. Method Forty eyes with attached posterior hyaloid were included in this prospective trial and randomized intraoperatively. Prior focal $(n=31)$ or panretinal $(n=25)$ laser coagulation was permitted. Group I ( $n=19$ patients) underwent
\end{abstract}

Meeting presentation: longterm results of vitrectomy for cystoid diabetic macular edema. Internal limiting membrane delamination vs posterior hyaloid removal. A prospective randomized trial. XXVIth Meeting of the Club Jules Gonin, St.Moritz, Switzerland, September 2008

Hans Hoerauf and Anne Brüggemann contributed equally to the manuscript.

\section{Financial Support None}

Conflict of Interest None of the authors have any financial / conflicting interests to disclose.

\footnotetext{
H. Hoerauf $(\square)$

University Eye Clinic, Georg-August-University,

Robert-Koch-Str. 40,

37075 Goettingen, Germany

e-mail: hanshoerauf@med.uni-goettingen.de

A. Brüggemann $\cdot$ J. Lüke $\cdot$ M. Müller

Dept. of Ophthalmology, University Clinics Schleswig-Holstein,

Campus Luebeck,

Luebeck, Schleswig-Holstein, Germany

M. Muecke

Hospital of the German Federal Armed Forces,

Ulm, Germany
}

surgical induction of posterior vitreous detachment (PVD), group II ( $n=20$ patients) PVD and removal of the ILM. Eleven patients with detached posterior hyaloid (group III) were not randomized, and ILM removal was performed. One eye had to be excluded from further analysis. Examinations included ETDRS best-corrected visual acuity (BCVA), fluorescein angiography (FLA) and OCT at baseline, 3 and 6 months postoperatively. Main outcome measure was BCVA at 6 months, secondary was foveal thickness.

Results Mean BCVA over 6 months remained unchanged in $85 \%$ of patients of group II, and decreased in $53 \%$ of patients of group I. Results were not statistically significant

\section{E. Stefánsson \\ Dept. of Ophthalmology, University of Iceland, \\ Reykjavik, Iceland}

\section{H.-P. Hammes}

Medical Clinic, University Mannheim,

Mannheim, Germany

C. Weiß

Clinical Trials Center,

Cologne, Germany 
different [group I: mean decrease log MAR 95\% CI (0.06; $0.32)$, group II: $(-0.02 ; 0.11)]$. OCT revealed a significantly greater reduction of foveal thickness following PVD with ILM removal [group I: mean change: $95 \%$ CI $(-208.95 \mu \mathrm{m}$; $-78.05 \mu \mathrm{m})$, group II: $(-80.90 \mu \mathrm{m}:+59.17 \mu \mathrm{m})]$.

Conclusion Vitrectomy, PVD with or without ILM removal does not improve vision in patients with DM type 2 and cystoid diabetic macular edema without evident vitreoretinal traction. ILM delamination shows improved morphological results, and appears to be beneficial in eyes with preexisting PVD.

Keywords Vitrectomy Posterior vitreous detachment . Internal limiting membrane . Optical coherence tomography . Cystoid diabetic macular edema

\section{Introduction}

In former investigations the vitreous and the vitreoretinal junction have been detected as a modulator of diabetic macular edema [1-7], and their precise role in the pathogenesis of diabetic maculopathy is widely debated [8]. Encouraged by reports showing that diabetic patients with posterior vitreous detachment (PVD) are less likely to develop macular edema [6] and that resorption may occur after a spontaneous PVD [2, 9], vitrectomy with removal of the posterior hyaloid was considered to be a therapeutic option in diffuse and cystoid edema, which often persists despite multiple laser treatments $[5,7,10]$. Lewis reported successful vitrectomy in selected patients with diabetic macular edema associated with visible posterior hyaloidal traction [5]. Furthermore, clinical improvement has been reported in eyes even when macular traction was stated to be absent [4, 11-15]. Tachi and Ogino reported on a prospective series of 58 eyes from 41 patients with visual acuity improvement in 53\% of cases. However, they performed a combined cataract extraction in most of them [13]. Several authors postulated a positive effect of additional peeling of the internal limiting membrane [ILM] in patients with diabetic macular edema refractory to laser photocoagulation [1,16-18]. Most reliable data on the efficacy of vitrectomy and PVD was published by Otani and Kishi and Stolba and coworkers, first reported on a non-randomized series of seven patients after unilateral vitrectomy with PVD and significant reduction of macular edema in comparison with the fellow eye [12]. Stolba et al. recently demonstrated in a prospective randomized trial that vitrectomy with ILM removal provides better visual and morphological results than the natural course [19]. Uncertainty remains as to whether vitrectomy and PVD alone is sufficient, or whether additional ILM delamination is beneficial. A prospective non-randomized study by Patel et al. indicated that ILM removal achieves an improved morphological outcome [20]. Gandorfer et al. showed a remarkable decrease of macular edema, with improvement of BCVA [1]. However, in most studies a reduction of macular edema did not consequently lead to a better visual acuity $[3,11]$.

Aim of this prospective randomized study was to evaluate the efficacy of surgical PVD and additional ILM removal in comparison to vitrectomy and PVD alone in typical diabetes mellitus type 2 patients with cystoid diabetic macular edema (CME) and without evident vitreomacular traction.

Due to the ILM removal, human material became available and was used to study sequestered factors, which could be involved in the pathogenesis of early diabetic retinopathy [21]. A further aim of the study was therefore to look for factors in the ILM possibly inducing vascular permeability changes.

\section{Methods}

\section{Demographic data}

The prospective randomized study population consisted of 50 patients (22 female, 28 male; 19 right eyes, 32 left eyes). Pars plana vitrectomies were performed at the Department of Ophthalmology, University Clinics Schleswig-Holstein, Campus Luebeck for cystoid diabetic macular edema between July 1999 and June 2003. Patients were divided into three groups: group I: surgical PVD, group II: surgical PVD and ILM removal, group III: preexisting PVD, vitrectomy and ILM removal. One eye of group I was excluded from further analyses because both eyes of one single patient had been randomized. Thirty eyes presented non-proliferative diabetic retinopathy (NPDRP) (group I: $73.68 \%$; group II: $60 \%$, group III: $36.36 \%$ ) and 20 eyes proliferative diabetic retinopathy (PDRP) (group I: $26.32 \%$; group II: $40 \%$; group III: $63.64 \%$ ). Nine out of these revealed active but mild neovascularizations of the disc or elsewhere; the others were quiescent. No fibrovascular tissue or traction was present. None of the patients showed a taut thickening of the posterior vitreous hyaloid by biomicroscopy, neither preoperatively, nor intraoperatively. Hard exudates in the macular region were present in 38 patients $(76 \%)$. None of the patients revealed an enlarged foveolar avascular zone on fluorescein angiography (FA). A detachment of the neurosensory retina from the retinal pigment epithelium or any sign of epiretinal membrane or vitreoretinal traction was ruled out by biomicroscopy and by optical coherence tomography (OCT).

At baseline, systemic hypertension controlled with medications was present in 12/19 patients in group I, 15/ 
20 patients in group II and 11/11 patients in group III. Insulin-dependent diabetes mellitus was present in 13/19 patients in group I, $15 / 20$ patients in group II and $9 / 11$ patients in group III. Mean $\mathrm{HbA}_{1 \mathrm{c}}$ levels were 7.59 in group I, 8.15 in group II and 7.85 in group III. The demographic data for each group are listed in Table 1.

Informed written consent was obtained for all patients, and the study protocol was approved by the local ethics committee.

\section{Study protocol}

The eligibility criteria for this study included cystoid macular edema unresponsive to one or more laser treatments (interval at least 3 months since last photocoagulation). Only patients with diabetes mellitus type 2 were enrolled in the study. Eyes with evident vitreomacular traction, macular non-perfusion spreading over an area of 2 disc diameters, optic atrophy, subfoveal plaques or vitreous hemorrhage were excluded, as well as eyes with dense cataract or other ocular diseases. Vitreomacular traction or epiretinal membranes were excluded by OCT. The main outcome measure was BCVA determined by ETDRS charts after 6 months. Secondary endpoint was foveal thickness measured by OCT after 6 months.

Intraoperatively, after a preexisting PVD was ruled out, the patient was either randomized to group I (surgical PVD only) or group II (surgical PVD and ILM removal). In all of them, a detachment of the posterior hyaloid was surgically induced. An additional ILM delamination was performed in group II. In 11 patients, the posterior hyaloid was intraoperatively found not to be attached to the retina, as had been presumed preoperatively. These eyes (22\%) were not randomized, but underwent ILM removal, and were followed by the regular examination regimen to evaluate the effect of ILM removal alone in patients with cystoid diabetic macular edema and preexisting PVD. They were summarized in group III. The participant flow chart according to the CONSORT statement [22] is shown in Fig. 1.

\section{Examinations}

Preoperatively and at 3 and 6 months postoperatively, all eyes underwent ETDRS visual acuity testing, a careful biomicroscopic evaluation, measurement of the foveal area thickness using optical coherence tomography (OCT II and III, Zeiss-Humphrey, Jena, Germany) and fluorescein angiography. All regular examinations were performed by the same examiner (MM). The visual acuity was evaluated following the guidelines of the study protocol of the "Early Treatment Diabetic Retinopathy Study Research Group" (ETDRS) [23]. The postoperative visual outcomes of the treatment groups were compared at 3 and 6 months.

Macular edema at baseline and during the follow-up was assessed by fluorescein angiography in terms of size and

Table 1 Demographic data and patients characteristics at baseline in the treatment groups

\begin{tabular}{|c|c|c|c|}
\hline Characteristics & Group I (PVD) & Group II (PVD+ILM-peeling) & Group III (ILM-peeling) \\
\hline Number of patients & 19 & 20 & 11 \\
\hline Gender $(n)$ : male/female & $10 / 9$ & $12 / 8$ & $6 / 5$ \\
\hline Mean age (years) & $66.0(\mathrm{SD} 8.96)$ & $63.55(\mathrm{SD} 7.14)$ & $70.0(\mathrm{SD} 6.23)$ \\
\hline Preop. visual acuity (logMAR, mean) & $0.59(\mathrm{SD} 0.23)$ & $0.59(\mathrm{SD} 0.23)$ & $0.65(\mathrm{SD} 0.27)$ \\
\hline Preoperative foveal thickness ( $\mu \mathrm{m}$, mean) & $425.25(\mathrm{SD} 83.25)$ & $442.13(\mathrm{SD} 83.73)$ & $478.9(\mathrm{SD} 134.86)$ \\
\hline \multicolumn{4}{|l|}{ Lens-status $(n)$ : } \\
\hline - phakic & 17 & 19 & 8 \\
\hline - pseudophakic & 2 & 1 & 3 \\
\hline \multicolumn{4}{|l|}{ Type of diabetic retinopathy $(n)$ : } \\
\hline - non-proliferative & $14(73.63 \%)$ & $12(60 \%)$ & $4(36.36 \%)$ \\
\hline - proliferative & $5(26.32 \%)$ & $8(40 \%)$ & $7(63.64 \%)$ \\
\hline \multicolumn{4}{|l|}{ Prior laser treatment $(n)$ : } \\
\hline - panretinal & 7 & 9 & 9 \\
\hline - focal/grid & 16 & 15 & 10 \\
\hline Hypertension $(n)$ & 12 & 15 & 11 \\
\hline $\mathrm{HbA}_{1 \mathrm{c}}$ level $(\%$, mean $)$ & 7.59 (SD 1.17) & $8.15(\mathrm{SD} 1.60)$ & $7.85(\mathrm{SD} 1.24)$ \\
\hline Serum creatine level ( $\mu$ mol/1, mean) & $78.33(\mathrm{SD} 28.10)$ & $78.00(\mathrm{SD} 20.84)$ & $108.00(\mathrm{SD} 41.15)$ \\
\hline Mean duration of diabetes mellitus (years, mean) & $14.75(\mathrm{SD} 6.56)$ & $12.67(\mathrm{SD} 5.40)$ & $16.56(\mathrm{SD} 9.55)$ \\
\hline Mean duration of macular edema (months, mean) & $10.40(\mathrm{SD} 8.32)$ & $10.29(\mathrm{SD} 7.14)$ & $22.22(\mathrm{SD} 20.20)$ \\
\hline Insulin dependent/non-insulin dependent $(n)$ & $13 / 6$ & $15 / 5$ & $9 / 2$ \\
\hline
\end{tabular}




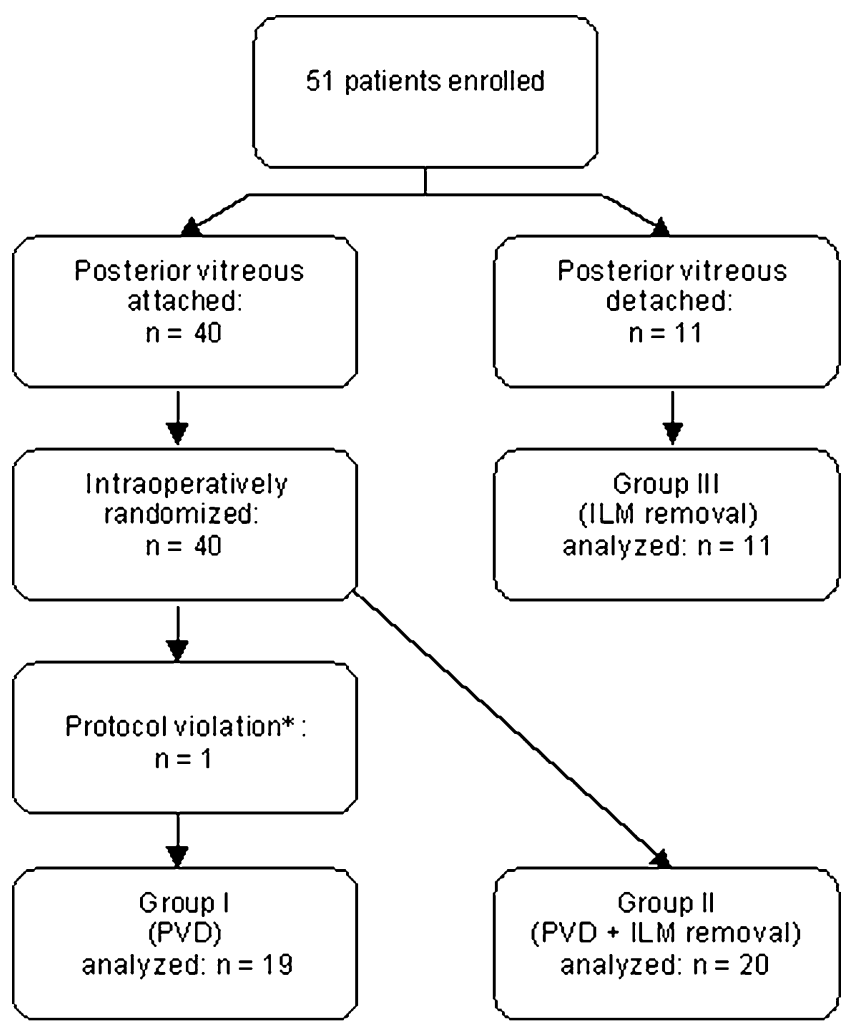

Fig. 1 Participant flow chart for each treatment group. $P V D=$ posterior vitreous detachment, ILM=internal limiting membrane, * two eyes of one single patient were randomized

intensity, and by OCT measurements to determine the foveal thickness, which was defined as the distance between the inner retinal surface and the retinal pigment epithelium at the central fovea. The fovea was scanned in a vertical direction.

To analyze the long-term outcome within this prospective study and to minimize the influence of cataract formation, we decided to perform a late follow-up examination of the patients, including BCVA and foveal thickness in addition to the study protocol. Long-term visual acuity values were obtained in part at our institution (ETDRS-VA) and in part by the referring ophthalmologists (Snellen VA). Longterm OCT thickness measurements could be obtained only in patients who were re-examined in our institution. Results are analyzed non-confirmatively.

\section{Surgical procedure}

After written informed consent was obtained, all operations were performed by a single surgeon $(\mathrm{HH})$ under general anaesthesia. A three-port pars plana vitrectomy was performed, and following core vitrectomy the posterior vitreous cortex was detached from the retina by suction over the optic nerve disc until the Weiss ring was identified. No triamcinolone was used for this manoeuver, in order to exclude a possible influence on the visual or morphological outcome.

In patients randomized to group II and in patients of group III, the ILM was removed using endgripping forceps. The first 12 patients (nine of group II and three patients of group III) underwent ILM removal without the use of dyes; in the subsequent 19 patients (eleven of group II and eight patients of group III) the ILM was stained with approximately $0.1 \mathrm{ml}$ of indocyanine green (ICG-Pulsion, Munich, Germany) dissolved in 5\% glucose in a concentration of $0.05 \%$ under constant irrigation with Ringer's solution. Immediately after application, dye remnants within the vitreous cavity or outside the central retina were washed out by suction using a flute needle or the ocutome. The ILM removal was extended over the whole edematous area to the vascular arcades, and was performed successfully in all eyes. The removed tissue was confirmed to be ILM by light microscopy, and in part further examined by immunohistochemistry. Intraoperatively, panretinal argon endolaser coagulation was performed in 11 of the 50 eyes (group I: three eyes, group II: five eyes, group III: three eyes) for the treatment of severe non-proliferative $(n=2)$ or proliferative diabetic retinopathy $(n=9)$. No focal or grid laser therapy was applied intraoperatively or within the follow-up period. Then, a careful examination of the retinal periphery by scleral indentation was carried out. Cryocoagulation was performed for peripheral iatrogenic tears $(n=10$; group I: three eyes, group II: five eyes, group III: two eyes). No endotamponade was intended, but was necessary for peripheral tears in ten eyes (gas: $n=4$, air: $n=6$ ).

\section{Statistical analysis}

Statistical analysis was performed using SAS (the SAS System; Release 9.1.3 SP 2; SAS Institute Inc., Cary, NC, USA) on Windows ${ }^{\circledR} 2000$ SP 4 (Microsoft Corp., Redmond, WA, USA). Values are given as frequency and percentage for qualitative, and mean and standard deviation for quantitative parameters respectively. 95\% confidence intervals were calculated using SAS PROC MEANS. Analyses of variance (with repeated measures for time: preoperatively and at 3 and 6 months) were performed to evaluate time and treatment effects on visual acuity (logMAR) and foveal thickness (OCT).

Immunhistochemistry of surgically excised ILM

Surgically removed ILM specimens of 24 patients were examined by immunhistochemistry. Twelve specimens were from patients of group II from our study, and 12 from idiopathic macular holes without diabetes mellitus served as control. The specimen were fixed in $4 \%$ buffered formalin and embedded in paraffin. Eight-micrometer $(8 \mu \mathrm{m})$-thick paraffin sections were cut and mounted onto silanized 
slides. ABC staining technique (Vector Laboratories/Linaris, PK-6200, Wertheim-Bettingen, Germany) was used. Slides were blocked in normal horse serum for 30 minutes at room temperature (RT). Primary antibodies were mouse monoclonal bFGF (Calbiochem, GF22, 1:100, Darmstadt, Germany), CML (Novo Nordisk, 2F8, 1:1000, Malmö, Sweden), methylglyoxal advanced glycation endproduct (AGE) antibody (Dr. M. Brownlee, Albert Einstein Institute of Medicine, New York, NY, USA, 1:500), tumor necrosis factor (TNF) alpha (Calbiochem, CN 654300, 1:100, Darmstadt, Germany), VEGF (Calbiochem, GF-25, 1:20, Darmstadt, Germany), plateletderived growth factor (PDGF)-B (Santa Cruz, sc-7878, 1:500, Heidelberg, Germany), and a goat polyclonal interleucin (IL)-6 (Santa Cruz, sc-1266, 1:100, Heidelberg, Germany). The slides were incubated for 1 hour at room temperature. After three washing steps for 5 minutes in PBS, the sections were incubated with the biotinylated secondary antibody (ABC Kit) for 1 hour at room temperature, followed by a second washing step. DAB as substrate was used for detection. Photos were taken by Leica digital camera and IM 50 software.

\section{Results}

Visual acuity

Group I After vitrectomy, mean BCVA in group I decreased from 20/80 (logMAR mean 0.59, SD 0.23 ) preoperatively to $20 / 100$ (logMAR mean 0.70, SD 0.28) at 3 months and 20/125 (logMAR mean 0.78, SD 0.34) at 6 months. BCVA improved more than 2 lines in one of 19 eyes $(5.3 \%)$, remained the same in nine eyes $(47.4 \%)$ and worsened in nine eyes (47.4\%).

Group II After vitrectomy, PVD and additional ILM delamination, mean BCVA (preoperatively 20/ 80 (logMAR mean 0.59, SD 0.23)) decreased to $20 / 100$ (logMAR mean 0.65, SD 0.28) at 3 months and 20/100 (logMAR mean 0.64, SD 0.21) at 6 months. BCVA improved more than 2 lines in one of 20 eyes $(5 \%)$, remained the same in 17 eyes (85\%) and decreased in two eyes $(10 \%)$.Comparing groups I and II, the functional outcome did not reveal a significant treatment effect, taking into account the repeated measures $(p=0.5492)$, but did show a significant time effect $(p=0.0040)$ : at 6 months, group I: mean decrease $0.19 \log$ MAR, SD $0.27,95 \%$ CI $[0.06 ; 0.32]$, group II: mean decrease $0.05 \log$ MAR, SD $0.14,95 \%$ CI $[-0.02 ; 0.11])$.
Group III In group III, patients having preexisting PVD, the mean preoperative BCVA was 20/100 $(\log$ MAR mean 0.65 , SD 0.27). The mean postoperative BCVA after vitrectomy and ILM removal was unchanged at 20/100 (logMAR mean 0.68 , SD 0.28) at 3 months, and increased to $20 / 80$ (logMAR mean 0.60 , SD 0.29 ) at 6 months postoperatively. BCVA improved more than 2 lines in three of 11 eyes $(27.3 \%)$, remained the same in six eyes $(54.5 \%)$ and worsened in two eyes (18.2\%). Since this group was not randomized, no confirmative comparison with the other groups was performed.

The functional results are presented in Fig. 2.

Foveal thickness measurement

Group I In patients of group I, mean preoperative foveal thickness was $425.25 \mu \mathrm{m}$ (SD $83.25 \mu \mathrm{m})$. Three months postoperatively, the mean foveal thickness in group I was $432.33 \mu \mathrm{m}$ (SD $77.26 \mu \mathrm{m})$, and at 3 months $415.2 \mu \mathrm{m}$ (SD $132.25 \mu \mathrm{m})$. There was no significant effect of time ( $p=0.9198)$.

Group II In patients of group II, mean preoperative foveal thickness was $442.13 \mu \mathrm{m}$ (SD $83.73 \mu \mathrm{m})$. Three months postoperatively, the foveal thickness in group II decreased to a mean of $352.62 \mu \mathrm{m}$ (SD $102.38 \mu \mathrm{m})$. Six months postoperatively, it

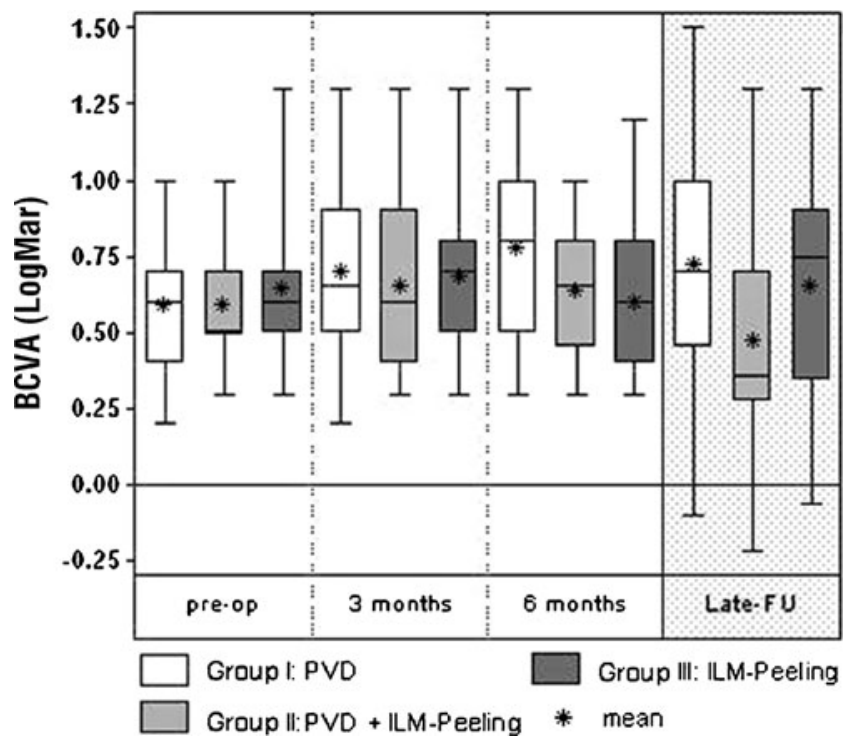

Fig. 2 Functional results of all groups, showing a decrease of bestcorrected visual acuity (BCVA) in group I with PVD only and stable BCVA in groups II and III with additional ILM removal. The influence of cataract progression has to be considered within the 6month follow-up period. Late follow-up BCVA values could be obtained only in part at our institution. $P V D=$ posterior vitreous detachment, $I L M=$ internal limiting membrane 
further decreased to a mean of $297.64 \mu \mathrm{m}$ (SD 139.92). This group showed a significant effect of time ( $p=0.0034)$. Comparing groups I and II, no significant treatment effect was detected $(p=$ 0.0871). However, in group II with ILM removal, an increased resolution of edema was found at 3 months [mean difference to preoperative value $-93.92 \mu \mathrm{m}$, SD $122.28 \mu \mathrm{m}, 95 \% \mathrm{CI}:(-167.81$; -20.03) compared to a mean difference of $-7.42 \mu \mathrm{m}$, SD $62.43 \mu \mathrm{m}$; 95\% CI: $(-47.08$; $32.25)$ in group I] and at 6 months [mean difference $-143.50 \mu \mathrm{m}$, SD $113.36 \mu \mathrm{m}, 95 \% \mathrm{CI}$ : $(-208.95 ;-78.05)$ compared to $-10.87 \mu \mathrm{m}, \mathrm{SD}$ $126.47 \mu \mathrm{m}, 95 \% \mathrm{CI}:(-80.90 ; 59.17)$ in group I].

Group III In group III patients having preexisting PVD, the preoperative mean foveal thickness was $478.90 \mu \mathrm{m}$ (SD $134.86 \mu \mathrm{m}$ ). Three months after vitrectomy and ILM removal the foveal thickness decreased to a mean of $308.13 \mu \mathrm{m}$ (SD $121.20 \mu \mathrm{m})$, and after 6 months to $296.60 \mu \mathrm{m}$ (SD $121.42 \mu \mathrm{m}$ ). As this group has not been randomized, no comparison with other groups was performed.

The anatomic results of all groups measured by OCT are presented in Fig 3.

Late follow-up-examination

Group I At a mean late follow-up of 56.01 months (SD 10.0 months) the functional data of 17 patients

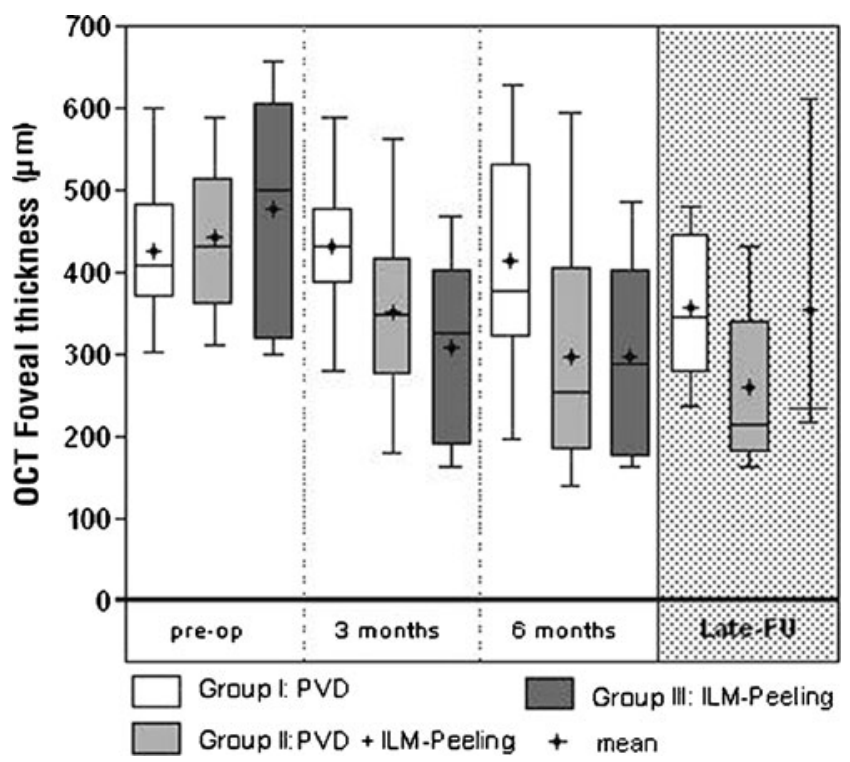

Fig. 3 Mean foveal thickness of treatment groups over the follow-up period, showing only a slight decrease of edema in group I and markedly better reabsorption in groups II and III with additional ILM removal. Late follow-up OCT values could be obtained only in some of the patients were available. Despite cataract extraction that had been performed in 13/16 phakic patients, mean BCVA remained unchanged at 20/100 (logMAR mean 0.72, SD 0.45), but mean foveal thickness of eight patients at 54.01 months (SD 8.0 months) further decreased to $357.0 \mu \mathrm{m}$ (SD $95.09 \mu \mathrm{m}$ ).

Group II At a mean late follow-up of 46.0 months (SD 17.0 months), cataract extraction had been performed in 13/19 phakic patients. BCVA of 17 patients at that time had decreased again to 20/63 (logMAR mean 0.47, SD 0.3). Correspondingly, mean foveal thickness of 12 patients measured at 49.0 months (SD 5.0 months) had slightly increased to $258.0 \mu \mathrm{m}$ (SD 96.50). Comparing the randomized treatment groups I and II at their late follow-up visit, the functional outcome in group II was favourable [group I: $\log$ MAR mean 0.16 , SD $0.39,95 \%$ CI: $(-0.05 ; 0.36)$ group II: $\operatorname{logMAR}$ mean $-0,09$, SD $0,32,95 \%$ CI: $(-0.25$; $0.08)$ ], but it has to be considered that only 17 patients of each of groups I and II were analyzed and in part the visual acuity values were obtained by the referring ophthalmologists (Snellen VA). The morphological outcome at the late follow-up visit again was favourable in group II: [group I: mean difference $-65.43 \mu \mathrm{m}$, SD $124.63 \mu \mathrm{m}, 95 \%$ CI: $(-180.69 ; 49.83)$, group II: $-184.11 \mu \mathrm{m}, \mathrm{SD}$ $141.78 \mu \mathrm{m}, 95 \%$ CI: (-293.09;-75.13)], but only in eight patients of group I and in 12 patients of group II an OCT measurement could be obtained. The mean decrease of foveal thickness in group II was $37.37 \%$ at the late visit [SD $24.82,95 \%$ CI: (18.29, $56.45)$ ], whereas in group I it was $13.53 \%$ at the late visit [SD 27.18, 95\% CI: $(-11.61 ; 38.67)]$.

Group III At the late follow-up visit at 48.0 months (SD 16.00) postoperatively, cataract extraction had been performed in all phakic patients $(n=8)$. BCVA of eight patients at that time was 20/100 ( $\log$ MAR mean 0.65 , SD 0.43 ). The mean foveal thickness measured only in three patients at 49.0 months (SD 8.00) was $354.67 \mu \mathrm{m}$ (SD $223.04 \mu \mathrm{m}$; single values: $235.0 \mu \mathrm{m}, 217.0 \mu \mathrm{m}$, $612.0 \mu \mathrm{m})$. The mean decrease was $28.92 \%$ [SD22.45, 95\% CI: $(-26.84,84.68)]$.

Subgroup analysis - indocyanine green

The first nine patients of group II and three patients of group III underwent ILM removal without the use of dyes. In 19 consecutive patients (11 of group II and eight of group III), an ICG-assisted peeling of the ILM was 
performed. Mean BCVA in the ICG group decreased slightly from $20 / 80$ [logMAR 0.59 , SD $0.19,95 \%$ CI: $(0.5 ; 0.68)]$ preoperatively to $20 / 80$ [ $\operatorname{logMAR} 0.61, \mathrm{SD}$ $0.21,95 \%$ CI: $(0.5 ; 0.71)]$ at 6 months and remained stable at the follow-up. Mean BCVA in the group without the use of ICG decreased from 20/80 [logMAR 0.64, SD 0.19, 95\% CI: $(0.44 ; 0.84)]$ preoperatively to $20 / 100$ [ $\log$ MAR 0.65 , SD $0.28,95 \%$ CI: $(0.47 ; 0.83)]$ at 6 months, and increased to $20 / 50$ [logMAR 0.36 , SD $0.34,95 \%$ CI: $(0.1 ; 0.63)$ ] at the late follow-up visit. Comparing both groups, ICG did not influence the functional outcome.

With regard to the morphological outcome, patients with the use of ICG revealed a decrease of mean foveal thickness from $483.94 \mu \mathrm{m}$ (SD $112.4 \mu \mathrm{m}, 95 \%$ CI: (442.02; 543.85)] preoperatively to $347 \mu \mathrm{m}$ [SD $116.85 \mu \mathrm{m}, 95 \%$ CI: $(284.74$; 409.26)] at 3 months, to $347.06 \mu \mathrm{m}$ [SD $128.51 \mu \mathrm{m}, 95 \% \mathrm{CI}$ : $(278.58 ; 415.54)]$ at 6 months and to $325 \mu \mathrm{m}$ [SD $161.02 \mu \mathrm{m}$, 95\% CI: (176.08; 473.92)] at the late follow-up visit. In patients without the use of ICG the mean foveal thickness preoperatively was $408.67 \mu \mathrm{m}$ [SD $77.21 \mu \mathrm{m}, 95 \% \mathrm{CI}$ : (349.31; 468.02)], at 3 months $299.4 \mu \mathrm{m}$ [SD $80.35 \mu \mathrm{m}, 95 \%$ CI: $(199.63 ; 399.17)], 197.5 \mu \mathrm{m}$ [SD $53.65 \mu \mathrm{m}, 95 \%$ CI: $(152.65 ; 242.35)]$ at 6 months and $235.63 \mu \mathrm{m}$ [SD $74.6 \mu \mathrm{m}$, 95\% CI: $(173.26 ; 297.99)]$ at the late follow-up visit.

Hard exudates

Group I The numbers of hard exudates increased in $7 / 15$ patients $(46.66 \%)$, remained the same in $3 / 15$ patients $(20 \%)$ and decreased in $5 / 15$ patients $(33.33 \%)$ at the 6-month visit in patients who underwent posterior vitreous detachment only.

Group II The number of hard exudates increased in 6/18 patients $(33 \%)$, remained the same in $2 / 18$ patients $(11 \%)$ and decreased in $10 / 18$ patients (56\%) 6 months postoperatively when an additional ILM delamination was performed.

Group III In group III with preexisting PVD, the number of hard exudates increased in $1 / 6$ patients $(16.5 \%)$, remained the same in $1 / 6$ patients $(16.5 \%)$ and decreased in $4 / 6$ patients $(67 \%) 6$ months after vitrectomy and ILM removal.ILM removal in groups II and III was qualitatively found to increase resorption rate of hard exudates.

Fluorescein angiography

Group I The extension of the edema enlarged in 5/19 patients $(26.32 \%)$, remained unchanged in $6 / 19$ patients $(31.58 \%)$ and diminished in $8 / 19$ patients $(42.11 \%)$ at 6 months. The intensity at that time increased in $7 / 19$ patients (36.84\%), remained unchanged in $1 / 19$ patients $(5.26 \%)$ and reduced in $11 / 19$ patients $(57.89 \%)$. The foveolar avascular zone (FAZ) had slightly enlarged in six patients and decreased in one patient.

Group II The extension of the edema enlarged in $2 / 20$ patients $(10 \%)$, remained unchanged in $8 / 20$ patients $(40 \%)$ and decreased in $10 / 20$ patients $(50 \%)$ at 6 months. The intensity at that time increased in $3 / 20$ patients $(15 \%)$, remained unchanged in $3 / 20$ patients $(15 \%)$ and reduced in $14 / 20$ patients $(70 \%)$. In two patients the FAZ slightly enlarged and diminished in three patients. Comparing groups I and II, additional ILM delamination did not qualitatively influence intensity or extension of edema.

Group III In group III with preexisting PVD, the extension of edema enlarged in $1 / 11$ patients $(9 \%)$, remained unchanged in $5 / 11$ patients $(45.5 \%)$ and decreased in $5 / 11$ patients $(45.5 \%)$ at 6 months. The intensity at that time increased in $2 / 11$ patients $(18 \%)$, remained unchanged in $2 / 11$ patients $(18 \%)$ and decreased in $7 / 11$ patients $(64 \%)$. In one patient the FAZ slightly enlarged, and in one patient FAZ decreased.

\section{Complications}

Iatrogenic tears were observed intraoperatively in ten patients ( $n=3$ in group I, $n=5$ in group II, $n=2$ in group III) and treated by cryocoagulation $(n=10)$ and air $(n=6)$ or gas endotamponade $(n=4)$.

Intraoperative iatrogenic opening or rupture of macular cysts was not observed in any patient. In 42/44 (95.45\%) phakic patients, cataract formation with nuclear sclerosis was observed within 6 months ( $n=15$ in group I, $n=19$ in group II, $n=8$ in group III).

Epiretinal membrane formation occured in one patient of group I and in one patient of group II. One patient of group II required re-vitrectomy and silicone oil tamponade for a dense postoperative vitreous hemorrhage. In three patients, a retinal detachment developed (group I: $n=1$, group II: $n=$ 1, group III: $n=1)$. These patients underwent successful revitrectomy with encircling band $(n=1)$, radial sponge $(n=$ $2)$, and gas endotamponade $(n=1)$.

Immunohistochemical results

ILMs of patients with diabetic maculopathy intensely stained for bFGF and methylglyoxal-type AGEs (Fig. 4). 
Fig. 4 Histologic section of a surgically removed internal limiting membrane from a patient of group II showing immunostainings for bFGF, methylglyoxal-type AGE and CML. Note that in comparison with ILM from patients with macular holes, there is no difference in CML deposition, but a substantial difference for methylglyoxal-type AGE, and, to a minor degree $\mathrm{bFGF}$

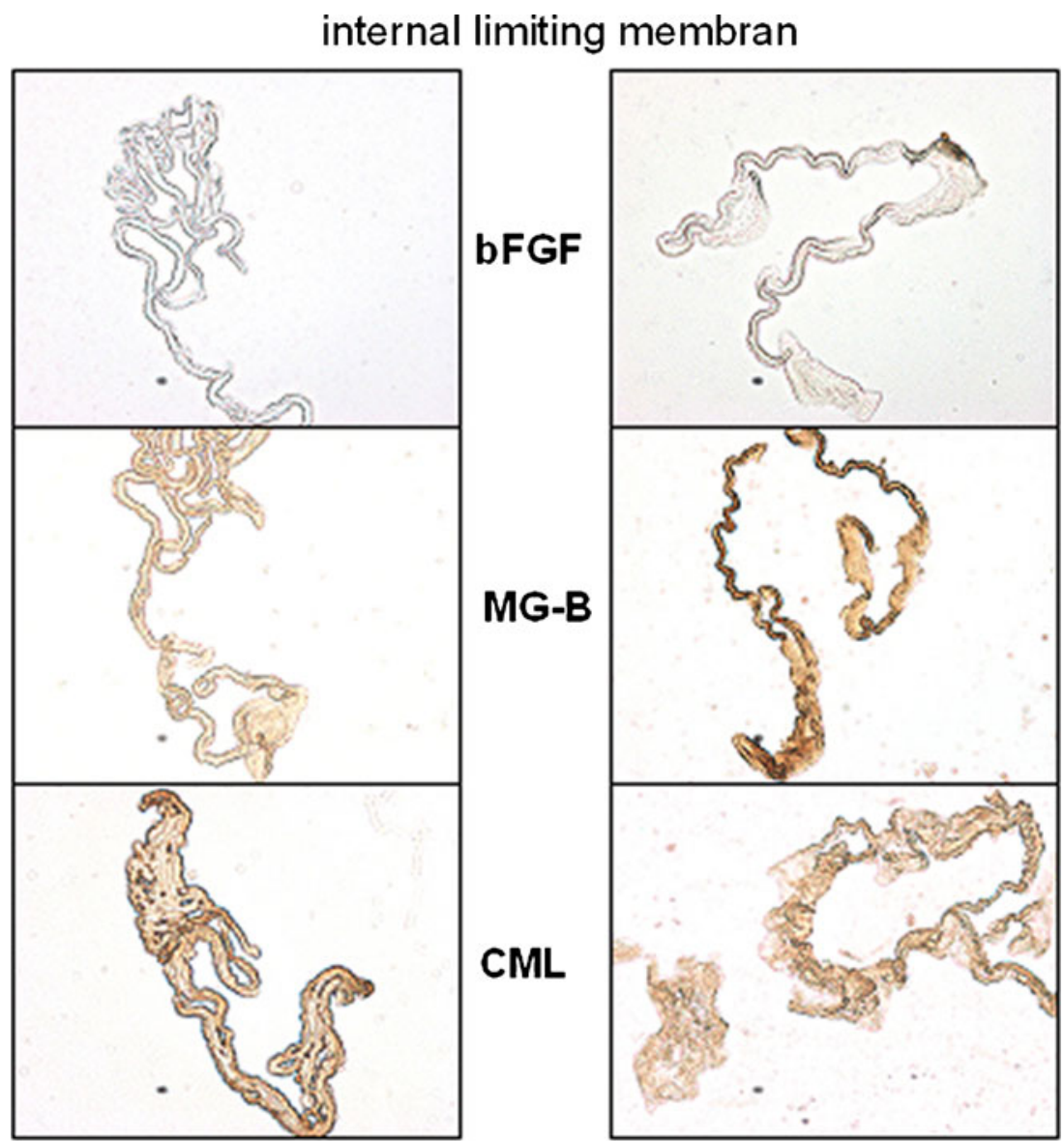

macular hole

diabetic maculopathy
In contrast, the glycoxidation marker CML was not different when compared with ILMs from patients with idiopathic macular holes. ILMs stained negatively for VEGF, PDGF-B, TNFalpha and IL-6.

\section{Discussion}

Few data from randomized controlled trials are available concerning the therapeutic effect of vitrectomy for diabetic macular edema. Recently, Stolba et al. reported a favourable outcome following vitrectomy and ILM peeling opposed to the natural course [19]. Yanyali et al. also observed a decrease in retinal thickness, without, however, significant improvement of visual acuity [15]. Uncertainty exists as to the necessity of ILM removal or whether PVD alone is sufficient. In a retrospective study, Stefaniotou et al. found that ILM peeling was beneficial in patients with diffuse diabetic macular edema [24]. In contrast, Bahadir and colleagues could not prove a beneficial effect of vitrectomy and ILM removal in comparison to vitrectomy alone [25]. No significant improvement of functional results in patients with diffuse diabetic macular edema was observed by Kumar et al. comparing vitrectomy with removal of ILM in contrast to grid laser photocoagulation [26].

The presented study provides the first randomized controlled trial data demonstrating a favourable effect of additional ILM removal in vitrectomy for cystoid diabetic macular edema in diabetes type 2 patients without evident vitreomacular traction, as shown in foveal thickness measurements by OCT. In the long term, while PVD alone slowly improves the anatomical results, it is markedly less effective than additional ILM removal. Although, the morphological results were substantial, visual results were unsatisfactory. Comparing the results of groups I and II, it can be stated that ILM removal shows the tendency of being beneficial in eyes with primarily attached vitreous in which a PVD was surgically induced analyzing the secondary endpoint of foveal thickness. However, identification of the posterior hyaloid by slit-lamp contact biomicroscopy is notoriously difficult, and even OCT can visualize the vitreoretinal interface only when it is partially detached or where the PVD is located within a few micrometers from the retinal surface. This was the reason why the randomization was performed intraoperatively, after a preexisting vitreous detachment was definitively 
ruled out by the surgeon. In group I, the surgeon relied only on the visualization of the Weiss ring during the PVD induction, based on careful observation and surgical experience. No triamcinolone was used for the identification of vitreous remnants, in order to exclude any influence of drug remnants on the resorption of the macular edema. An incorrect intraoperative judgement of the posterior vitreous may have influenced the results. Even during surgery, it may be hard to distinguish a PVD from vitreoschisis, especially in patients with diabetic retinopathy. In those 20 eyes where ILM removal was performed, it is extremely unlikely that the posterior vitreous was still attached, especially in patients in whom ICG was used for ILM removal, since dyes such as ICG facilitate visualization of vitreous remnants.

Moreover, our results suggest that even when a spontaneous PVD is present (group III), vitrectomy with ILM removal may provide favourable morphological results. It has to be emphasized that this group III was not randomized, and that the number of included patients in all groups was small. Due to this limiting drawback of the present study, subgroup evaluation was complex, and the conclusions must be looked at with caution. Nevertheless, the study design was prospective.

The most distinct effect of our study seen on fluorescein angiography was not in the decrease in edema size but rather the reduction of staining intensity. This parameter is difficult to quantify and OCT has become an important tool, providing detailed morphologic information and allowing the detection of macular edema with a sensitivity of $89 \%$ and a specificity of $96 \%[1,27,28]$. However, the resolution available in OCT systems is not high enough to distinguish between intra- and extracellular edema.

The exact pathomechanism of ILM removal on diabetic macular edema is currently unknown. The ILM, which is the basement membrane of the Müller cells, may act as a diffusion barrier. Recent findings of a comparative histopathological study of surgically removed ILM showed that the ILMs in patients with diabetic macular edema were significantly thicker (mean thickness: $4.8 \pm 1.6 \mu \mathrm{m}$ ), revealing a larger amount of cellular elements on the vitreous side than in patients with macular holes $(1.8 \pm 0.6 \mu \mathrm{m})$ [1, 29]. This thickened ILM may act as a barrier, decreasing transretinal fluid movement. After removal of this diffusion barrier, the disturbance of transretinal fluid movement may be in part restored and contribute to a resolution of the macular edema [1]. Also, Radetzky et al. speculated about a pseudomembrane formed by the endplates of Müller cells exerting a barrier function [30]. Intraretinal structural damage in chronic cystoid edema may further influence the diffusion properties.

It is also possible that the thickened ILM may reduce the diffusion of oxygen from the vitreous cavity to the retina following vitrectomy. Stefánsson et al. have suggested that fluid currents in the vitreous cavity following vitrectomy of PVD transport oxygen from well-perfused areas of the retina to hypoxic areas such as in diabetic macular edema [31, 32]. The diffusion of oxygen from the fluid in the vitreous cavity into the retina would be retarded by a thickened ILM.

Another theory is that the repair mechanisms of Müller cells may be activated by delamination of the ILM [33]. Also, the absence of the vitreous gel would increase the transport of cytokines, such as VEGF, from the retina into the vitreous cavity, and the absence of ILM would further speed up this clearance of cytokines from the retina [8].

The efficacy of ILM delamination may be caused by the removal of a growth factor reservoir which may have accumulated in the ILM and in cellular elements on its vitreous side. A recent study showed that vitreous remnants may be present after surgical vitreous separation [34]. Histopathologic correlations by Gandorfer et al. [35] and Matsunaga et al. [29] support the theory that ILM delamination allows a more complete removal of vitreous elements.

As Fig. 4 demonstrates, CML and AGEs could be detected within removed ILM specimens of patients with diabetic macular edema of groups II and III, in contrast to those gained during macular hole surgery. AGEs have been implicated in the primary pathogenesis of diabetic vascular damage that ultimately leads to progressive vascular occlusions, as in diabetic maculopathy. Furthermore, AGEs can enhance VEGF production and thereby induce permeability changes. The finding of bFGF in the ILMs of diabetic eyes supports previous data showing bFGF deposits in the inner limiting membranes of retinae from diabetic patients [36], and of experimentally diabetic animals [37]. It is worth noting that bFGF is an important repair/survival factor for retinal glia suggesting that our finding is consistent with increased repair, rather than a sign of damage. The glycoxidation product CML is present in a variety of conditions associated with increased oxidative or glycative stress, in particular in the eye [38-40]. The finding that methylglyoxal-type AGE is found in membranes representing the endfeet of Müller cells is of particular clinical interest, given the present debate concerning the role of neuroglia in the course and pathogenesis of diabetic retinopathy, and the possible role of these specific AGEs in the link between metabolic stress and transcriptional regulation of genes in Müller cells [41]. AGEs can enhance VEGF production, and thereby may induce permeability changes.

Compared to the promising anatomical findings, the functional results in our study are less beneficial. The additional ILM delamination still seemed to stabilize visual acuity with an unchanged or better BCVA in $90 \%$ of patients, despite development of a marked complicating cataract in most patients within the follow-up period. There was a statistically significant effect over time, revealing higher 
visual deterioration of BCVA in group I without ILM removal [mean decrease of $0.19 \log$ MAR, SD $0.27,95 \% \mathrm{CI}(0.06 ; 0.32$ $\operatorname{logMAR}$ )] than in group II [mean decrease $0.05 \log$ MAR, SD $0.14,95 \%$ CI $(-0.02 ; 0.11)]$ but without treatment difference. To rule out the influence of cataract formation at the end of the study, we decided to additionally determine long-term visual and morphological results. This extension of the study was not planned in the initial study protocol. Most of the patients had undergone cataract extraction in the meantime, but surprisingly there was no remarkable change among the groups. Only group II showed improved VA results compared to baseline; groups I and III remained stable.

Our limited functional results in group I stand in contrast to recently published studies about vitrectomy without ILM peeling in diabetic macular edema. La Heij et al. reported on the resolution of diabetic macular edema in 21 of 21 eyes [11], and Tachi and Ogino in 57 of 58 eyes [13]. However, both studies based their findings on fluorescein angiography and biomicroscopy, which makes an objective measurement of retinal thickness difficult if not impossible. Furthermore, the study results of La Heij are completely based on follow-up examinations from referring ophthalmologists only, which raises doubt about the standardization of measurement conditions. Ikeda et al. performed vitrectomy on three eyes of two patients in which evidence of macular traction was specifically noted to be absent. They observed resolution of macular edema with improvement of visual acuity in all eyes [4]. In a recent retrospective study, Rosenblatt et al. also reported significant improvement of visual acuity and diminution of retinal thickness in patients with refractory diabetic macular edema without taut posterior hyaloid [18]. The preexisting compromised retinal microvasculature in diabetic eyes is likely to be more vulnerable to exsudation when subjected to traction. Therefore, it is understandable that vitrectomy and posterior vitreous separation was beneficial in selected patients with diabetic macular edema when the hyaloid has been judged to be taut $[5,7,10]$. However, the patients in our study represent the typical situation in most elderly diabetic mellitus type 2 patients where no clinically visible vitreo-macular traction is present. Although there has been a considerable number of studies implicating an important role of the posterior hyaloid in the pathogenesis of diabetic macular edema [11-13, 16], we did not find any effect of posterior hyaloid detachment alone in our study.

One possible cause for the discrepancy of those studies in comparison with our results is the longer history of macular edema resulting in cystoid changes in a high number of patients, and the selection of diabetic mellitus type 2 patients in our patient series, which may have had a negative impact on photoreceptor recovery in this elderly study population. The presence of cystoid macular edema in our study population is a clinical sign for the chronic nature of the edema and longstanding intraretinal diabetic changes with irreversible photoreceptor damage. Moreover, the limited functional results in our study may have been influenced by bad systemic glucose control ( $\mathrm{HbA}_{1 \mathrm{c}}: 7.59-8.15$, see Table 1) [42].

Futhermore, visual outcome might have been adversely affected by one or more prior grid laser photocoagulations, treatment which was an inclusion criterion for our study. During surgery in 11 patients, panretinal argon endolaser coagulation due to severe non-proliferative or proliferative diabetic retinopathy was performed, in ten patients peripheral cryocoagulation and endotamponade for peripheral iatrogenic tears. These findings had no statistical consequence on functional or anatomic outcomes. However, as group sizes are fairly small, we cannot rule out an additional impact.

Dillinger and Mester also observed a discrepancy between the anatomical and functional outcome; however, they still reported a significant visual improvement of $43 \%$, gaining 2 lines of vision or more. In accordance with our positive results in groups II and III, the authors reported a decrease in macular thickening in 55 of 60 eyes after vitrectomy and ILM removal in chronic diabetic macular edema [16]. Our study was performed prospectively, but the quality of follow-up was limited due to postoperative examinations by the referring ophthalmologists and loss of standardized conditions.

Recently, in a prospective non-randomized study, Patel et al. confirmed the beneficial effect of ILM removal on morphological outcome, but also found limited visual improvement [20]. They suspected that the normal Müller cell physiology may be altered after ILM removal, which may explain the lack of significant improvement in vision despite a marked resolution of macular edema. We could not show a significant effect of ILM removal; however, a tendency towards improved results after PVD with additional ILM removal could be seen.

Since ICG should allow a more complete ILM removal, better morphological functional results could be expected in the subgroup of patients in which it was used for staining. However, in our study ICG seemed to affect visual and morphological outcome negatively. Avci et al. reported about spontaneous ILM detachment from the neurosensory retina after ICG-assisted ILM peeling in patients with diabetic macular edema, using $0.25 \%$ ICG solution [43] for 1 minute after total fluid-air exchange. We used a markedly lower concentration of $0.05 \%$ dissolved in glucose under constant irrigation. But the observed tendency to poor results using ICG corresponds with other reports on possible toxicity of ICG. Brilliant Peel ${ }^{\circledR}$ was not available at that time.

The complication spectrum and rate is comparable to other macular surgery studies, with a higher rate of retinal tears and retinal detachments after surgically induced posterior vitreous detachment.

In conclusion, our study underlines the efficacy of PVD with additional ILM removal on resorption of 
cystoid diabetic macular edema, even without the presence of vitreomacular traction. Improvement or at least stabilization most likely can only be achieved when the macular edema is reduced. A further visual deterioration such as in group I is very likely if the macular edema persists. A negative influence of ILM removal on the visual outcome could not be proven by our data. The procedure seemed to stabilize visual acuity, and therefore this treatment option might be considered in earlier stages of the disease to preserve vision at higher levels. However, it has to be kept in mind that the study design did not allow the assessment of the efficacy of vitrectomy in diabetic macular edema against the natural course or grid laser coagulation, which is the current gold standard. Whether it is preferable to perform this procedure as a first-line treatment for diffuse diabetic macular edema instead of grid laser coagulation must be evaluated in another trial. New treatment modalities such as intravitreal triamcinolone acetonide [44-46] or antiVEGF drugs [47, 48] are promising, but recurrences and the requirement of multiple re-injections are the major backdraw of presently available drugs. As our late analysis suggests, vitrectomy and ILM removal is able to stabilize visual and morphological results also on the longterm.

Open Access This article is distributed under the terms of the Creative Commons Attribution Noncommercial License which permits any noncommercial use, distribution, and reproduction in any medium, provided the original author(s) and source are credited.

\section{References}

1. Gandorfer A, Messmer EM, Ulbig MW, Kampik A (2000) Resolution of diabetic macular edema after surgical removal of the posterior hyaloid and the inner limiting membrane. Retina 20 (2):126-133

2. Hikichi T, Fujio N, Akiba J, Azuma Y, Takahashi M, Yoshida A (1997) Association between the short-term natural history of diabetic macular edema and the vitreomacular relationship in type II diabetes mellitus. Ophthalmology 104(3):473-478

3. Ikeda T, Sato K, Katano T, Hayashi Y (1999) Vitrectomy for cystoid macular oedema with attached posterior hyaloid membrane in patients with diabetes. Br J Ophthalmol 83(1):12-14

4. Ikeda T, Sato K, Katano T, Hayashi Y (2000) Improved visual acuity following pars plana vitrectomy for diabetic cystoid macular dema and detached posterior hyaloid. Retina 20(2):220-222

5. Lewis H, Abrams GW, Blumenkranz MS, Campo RV (1992) Vitrectomy for diabetic macular traction and edema associated with posterior hyaloidal traction. Ophthalmology 99(5):753759

6. Nasrallah FP, Jalkh AE, an Coppenolle F, Kado M, Trempe CL, McMeel JW, Schepens CL (1998) The role of the vitreous in diabetic macular edema. Ophthalmology 95(10):1335-1339

7. Pendergast SD, Hassan TS, Williams GA, Cox MS, Margherio RR, Ferrone PJ, Garretson BR, Trese MT (2000) Vitrectomy for diffuse diabetic macular edema associated with a taut premacular posterior hyaloid. Am J Ophthalmol 130(2):178-186

8. Stefánsson E (2009) Physiology of vitreoussurgery. Graefes Arch Clin Exp Ophthalmol 247(2):147-163

9. Hartley KL, Smiddy WE, Flynn HW Jr, Murray TG (2008) Pars plana vitrectomy with internal limiting membrane peeling for diabetic macular edema. Retina 28(3):410-419

10. Harbour JW, Smiddy WE, Flynn HW, Rubsamen PE (1996) Vitrectomy for diabetic macular edema associated with a thickened and taut posterior hyaloid membrane. Am J Ophthalmol 121(4):405-413

11. La Heij EC, Hendrikse F, Kessels AG, Derhaag PJ (2001) Vitrectomy results in diabetic macular oedema without evident vitreomacular traction. Graefes Arch Clin Exp Ophthalmol 239 (4):264-270

12. Otani T, Kishi S (2002) A controlled study of vitrectomy for diabetic macular edema. Am J Ophthalmol 134(2):214-219

13. Tachi N, Ogino N (1996) Vitrectomy for diffuse macular edema in cases of diabetic retinopathy. Am J Ophthalmol 122(2):258-260

14. Aboutable T, Kalvodova B (2005) Vitrektomie beim diabetischen zystoiden Makulaödem - Ergebnisse von 72 Fällen. Klin Monatsbl Augenheilkd 222:643-648

15. Yanyali A, Horozoglu F, Celik E, Nohutcu AF (2007) Long-term outcomes of pars plana vitrectomy with internal limiting membrane removal in diabetic macular edema. Retina 27(5):557-566

16. Dillinger P, Mester U (2004) Vitrectomy with removal of the internal limiting membrane in chronic diabetic macular oedema. Graefes Arch Clin Exp Ophthalmol 242(8):630-637

17. Recchia FM, Ruby AJ, Carvalho Recchia CA (2005) Pars plana vitrectomy with removal of the internal limiting membrane in the treatment of persistent diabetic macular edemal. Am J Ophthalmol 139(3):447-454

18. Rosenblatt BJ, Shab GK, Sharma S, Bakal J (2005) Pars plana vitrectomy with internal limiting membranectomy for refractory diabetic macular edema without a taut posterior hyaloid. Graefes Arch Clin Exp Ophthalmol 243:20-25

19. Stolba U, Binder S, Gruber D, Krebs I, Aggermann T, Neumaier B (2005) Vitrectomy for persistent diffuse diabetic macular edema. Am J Ophthalmol 140:295-301

20. Patel JI, Hykin PG, Schadt M, Luong V, Fitzke F, Gregor ZJ (2006) Pars plana vitrectomy with and without peeling of the inner limiting membrane for diabetic macular edema. Retina 26:513

21. Kaiser PK (2006) Antivascular endothelial growth factor agents and their development: therapeutic implications in ocular diseases. Am J Ophthalmol 142(4):660-668

22. Moher D, Schulz KF, Altman DG, Consort Group (2001) The CONSORT statement: revised recommendations for improving the quality of reports of parallel-group randomised trials. Lancet 357:1191-1194

23. Early Treatment Diabetic Retinopathy Study Group (1987) Treatment techniques and clinical guidelines for photocoagulation of diabetic macular edema. Early treatment diabetic retinopathy study report number 2. Ophthalmology 94(7):761-774

24. Stefaniotou M, Aspiotis M, Kalogeropoulos C, Christodoulou A, Psylla M, Ioachim E, Alamanos I, Psilas K (2004) Vitrectomy results for diffuse diabetic macular edema with and without inner limiting membrane removal. Eur J Ophthalmol 14:137-143

25. Bahadir M, Ertan A, Mertoglu O (2005) Visual acuity comparison of vitrectomy with and without internal limiting membrane removal in the treatment of diabetic macular edema. Int Ophthalmol 26(1-2):3-8

26. Kumar A, Sinha S, Azad R, Sharma YR, Vohra R (2007) Comparative evaluation of vitrectomy and dye-enhanced ILM peel with grid laser in diffuse diabetic macular edema. Graefes Arch Clin Exp Ophthalmol 245(3):360-368 
27. Hoerauf H, Wirbelauer C, Scholz C, Engelhardt R, Koch P, Laqua H, Birngruber R (2000) Slit-lamp-adapted optical coherence tomography of the anterior segment. Graefes Arch Clin Exp Ophthalmol 238(1):8-18

28. Goebel W, Kretzchmar-Gross T (2002) Retinal thickness in diabetic retinopathy: a study using optical coherence tomography (OCT). Retina 22(6):759-767

29. Matsunaga N, Ozeki H, Hirabayashi Y, Shimada S, Ogura Y (2005) Histopathologic evaluation of the internal limiting membrane surgically excised from eyes with diabetic maculopathy. Retina 25:311-316

30. Radetzky S, Walter P, Fauser S, Koizumi K, Kirchhof B, Joussen AM (2004) Visual outcome of patients with macular edema after pars plana vitrectomy and indocyanine green-assisted peeling of the internal limiting membrane. Graefes Arch Clin Exp Ophthalmol 242:273-278

31. Stefánsson E, Novack RL, Hatchell DL (1990) Vitrectomy prevents hypoxia in branch retinal vein occlusion. Invest Ophthalmol Vis Sci 31:284-289

32. Stefánsson E (2001) Therapeutic effects of retinal laser treatment and vitrectomy. A theory based on oxygen and vascular physiology. Acta Ophthalmol Scand 79:435-440

33. Uckermann O, Uhlmann S, Pannicke T, Francke M, Gamsalijew R, Makarov F, Ulbricht E, Wiedemann P, Reichenbach A, Osborne NN, Bringmann A (2005) Ischemia-reperfusion causes exudative detachment of the rabbit retina. Invest Ophthalmol Vis Sci 46(7):2592-2600

34. Gandorfer A, Rohleder M, Grosselfinger S, Haritoglou C, Ulbig M, Kampik A (2005) Epiretinal pathology of diffuse diabetic macular edema associated with vitreomacular traction. Am J Ophthalmol 139:638-652

35. Sonada KH, Sakamoto T, Enaida H, Miyazaki M, Noda Y, Nakamura T, Ueno A, Yokoyama M, Kubota T, Ishibashi T (2004) Residual vitreous cortex after surgical posterior vitreous separation visualized by intravitreous triamcinolone acetonide. Ophthalmology 111:226-230

36. Hanneken A, Juan E Jr, Lutty GA, Fox GM, Schiffer S, Hjelmeland LM (1991) Altered distribution of basic fibroblast growth factor in diabetic retinopathy. Arch Ophthalmol 109:1005-1011

37. Hammes HP, Weiss A, Hess S, Araki N, Horiuchi S, Brownlee M, Preissner KT (1996) Modification of vibronectin by advanced glycation alters functional properties in vitro and in the diabetic retina. Lab Invest 75:325-338

38. Hammes HP, Alt A, Niwa T, Clausen JT, Bretzel RG, Brownlee M, Schleicher ED (1992) Differential accumulation of advanced glycation endprocucts in the course of diabetic retinopathy. Diabetologica 42:728-736

39. Hammes HP, Hoerauf H, Alt A, Schleicher E, Clausen JT, Bretzel RG, Laqua H (1990) N(epision)(carboxymethyl)lysine and the AGE receptor RAGE colocalize in age-related macular degeneration. Invest Ophthalmol Vis Sci 40:1855-1859

40. Augustin AJ, Spitznas M, Koch F, Grus F, Böker T (1995) Indications of oxidative tissue damage and inflammatory activity in epiretinal membranes of proliferative diabetic retinopathy, proliferative vitreoretinopathy and macular pucker. Ger J Ophthalmol 4:47-51

41. Yao D, Taguchi T, Matsumura T, Pestell R, Edelstein D, Giardino I, Suske G, Ahmed N, Thornalley PJ, Sarthy VP, Hammes HP, Brownlee M (2006) Methylglyoxal modification of mSin3A links altered glycolysis to angiopoietin-2 transcription: A novel mechanism for regulating gene expression. Cell 124:275-286

42. Nishikawa T, Edelstein D, Xue LD, Yamagishi S, Matsumura T, Kaneda Y, Yorek MA, Beebe D, Oates PJ, Hammes HP, Giardino I, Brownlee M (2000) Normalizing mitochondrial superoxide production blocks three pathways of hyperglycaemic damage. Nature 404:787-790

43. Avci R, Avci B, Kaderli B, Cavusoglu I (2004) A new surgical approach for indocyanine green-assisted internal limiting membrane peeling. Ophthalmic Surg Lasers Imaging 35:292-297

44. Jonas JB, Spandau UH, Kamppeter BA, Vossmerbaeumer U, Harder B (2006) Follow-up after intravitreal triamcinolone acetonide for diabetic macular edema. Eur J Ophthalmol 16 (4):566-572

45. Gillies MC, Sutter FK, Simpson JM, Larsson J, Ali H, Zhu M (2006) Intravitreal triamcinolone for refractory diabetic macular edema: two-year results of a double-masked, placebo-controlled, randomized clinical trial. Ophthalmology 113(9):1533-1538

46. Jonas JB, Kamppeter BA, Harder B, Vossmerbaeumer U, Sauder G, Spandau UH (2006) Intravitreal triamcinolone acetonide for diabetic macular edema: a prospective, randomized study. J Ocul Pharmacol Ther 22(3):200-207

47. Cunningsham ET Jr, Adamis AP, Altaweel M, Aiello LP, Bressler NM, D'Amico DJ, Goldbaum M, Guyer DR, Katz B, Patel M, Schwartz SD, Macugen Diabetic Retinopathy Study Group (2005) A phase II randomized double-masked trial of pegaptanib, an antivascular endothelial growth factor aptamer, for diabetic macular edema. Ophthalmology 112(10):1747-1757

48. Haritoglou C, Kook D, Neubauer A, Wolf A, Priglinger S, Strauss R, Gandorfer A, Ulbig M, Kampik A (2006) Intravitreal bevacizumab (Avastin) therapy for persistent diffuse diabetic macular edema. Retina 26(9):999-1005 